\title{
An AGS Experiment to Test Bunching for the Proton Driver of the Muon Collider
}

\author{
J. Norem*, C. Ankenbrandt, K-Y. Ng, M. Popovic, Z. Qian \\ Fermilab. Batavia IL. 60510
}

\author{
L. A. Ahrens, M. Brennan, V. Mane, T. Roser, \\ D. Trbojevic, W. van Asselt \\ Brookhaven National Laboratory, Upton NY 1197.3
}

\begin{abstract}
The proton driver for the muon collider must produec shore pulses of protons in order to facilitace muon cooling and operation with polarized heams. In order to test mechods of producing these bunches we have operated the AGS near transition and studied procectures which involved moving the transition energy $y_{1}$ to the hean encrgy. We were able in produce stable bunches with RMS widths of $\sigma=2.2-2.7 \mathrm{~ns}$ for lungizudinal bunch areas of $-1.5 \mathrm{~V}-\mathrm{s}$. in addition to making measurements of the lowest two orders of the momenturn compaclion factor.
\end{abstract}

\section{INTRODUCTION}

Muon collider designs $(1,2)$ zequire that the pions from which muons are produced are generated in a short bunch, both to facilitate subsequent cooling and to improve the separation between polarization states. Calculations done for the Feasibility Study imply that the required initial rms bunch length from the proton driver (3) is $\sigma-1-2$ ns, more than a factor of three shorter than the natural bunch length in the Brookhaven AGS (4). Since the proton driver contemplated for the muon collider could have its extraction energy near the natural transition energy, and would probably use a flexible momentum compaction (FMC) (5) lattice with easily adjustable transition energy, we have looked at options which could make use of transition in bunching. This paper summarizes simulations and experiments on the AOS near transition.

Bunching near transition is complicated by the nonlinearity in phase shear which introduces nonlinear motion in symchrotron space, as well as the small value of $|\eta|$ which reduces the synchrotron frequency, making these nonlinearities more prominent. Following reference (6), the expression for the circumference

$$
C(p)=C_{0}\left[1+\alpha_{0} \delta\left(1+\alpha_{1} \delta+\alpha_{2} \delta^{2}\right)+\ldots\right] .
$$




\section{DISCLAIMER}

This report was prepared as an account of work sponsored by an agency of the United States Government. Neither the United States Government nor any agency thereof, nor any of their employees, make any warranty, express or implied, or assumes any legal liability or responsibility for the accuracy, completeness, or usefulness of any information, apparatus, product, or process disclosed, or represents that its use would not infringe privately owned rights. Reference herein to any specific commercial product, process, or service by trade name, trademark, manufacturer, or otherwise does not necessarily constitute or imply its endorsement, recommendation, or favoring by the United States Government or any agency thereof. The views and opinions of authors expressed herein do not necessarily state or reflect those of the United States Government or any agency thereof. 


\section{DISCLAIMER}

Portions of this document may be illegible in electronic image products. Images are produced from the best available original document. 
where $d p / p=\delta$, and $C_{0}$ is the median circumference $(\delta=0)$, and the $\alpha$ 's are various orders of the momentum compaction. One also defines the slip factor as

$$
\eta \equiv \Delta T / T / \delta,
$$

giving the transition gamma,

$$
\gamma_{l}(\delta)=\gamma_{\text {to }}\left\{1-\left(\alpha_{1}+0.5-\alpha_{0} / 2\right) \delta+O\left(\delta^{2}\right)\right\},
$$

the first order momentum compaction is therefore

$$
\alpha_{1}=-1 / \gamma_{10} \mathrm{~d} \gamma_{\tau} / \mathrm{d} \delta-1 / 2+\alpha_{0} / 2 .
$$

Predictions of MAD (7) for the AGS are shown in Figure 1.

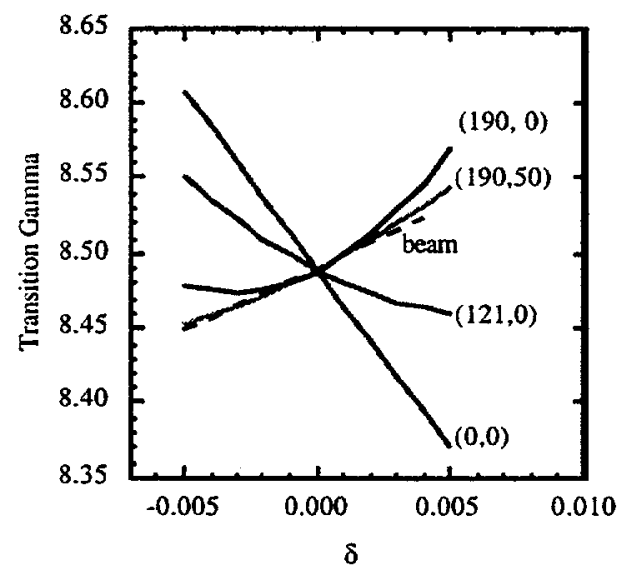

FIGURE 1. Predicted momentum dependence of $\gamma_{1}$ as a function of sextupole and octupole settings $\left(\mathrm{I}_{\mathrm{s}}, \mathrm{I}_{\mathrm{O}}\right)$.

\section{OPERATION OF THE AGS}

The operating mode of the AGS is shown in Figure 2. 'The maximum beam energy was reduced from the normal $28 \mathrm{GeV}$ to around $7 \mathrm{GeV}$ by shortening the acceleration period, and the $\gamma$ jump system (8) was modified to give a short flattop period before the transition energy dropped. The beam was flattopped for $300 \mathrm{~ms}$ before the magnet guide field was raised slightly and then ramped down. One bunch was injected from the booster at about $0.08 \mathrm{sec}$ into the acceleration cycle, when $\mathrm{d} B / \mathrm{d} t-0.4 \mathrm{~T} / \mathrm{s}$, and the beam was in the machine for about $500 \mathrm{~ms}$ total. The rf cavities were operated at $\sim 340$ $\mathrm{kV} / \mathrm{turn}$ while there was beam. The bunch current was $3-5 \times 10^{12}$ in a single bunch, and the bunch area was measured to be $1.6-1.5 \mathrm{eV}$-s.

Previous work had shown that the $\gamma_{t}$ jump system caused significant changes in the nonlinear momentum compaction factor $\alpha_{1}$, the measured maximum dispersion and the momentum aperture. Although three sextupole families are present in the ring, the 
locations of these sextupoles are such that these lattice changes are difficult to tune out both before and after the transition energy is moved. In order to evaluate the magnitude of these effects we remeasured the transition energy as a function of radial position by measuring beam loss as a function of phase-switch delay time and sextupole setting

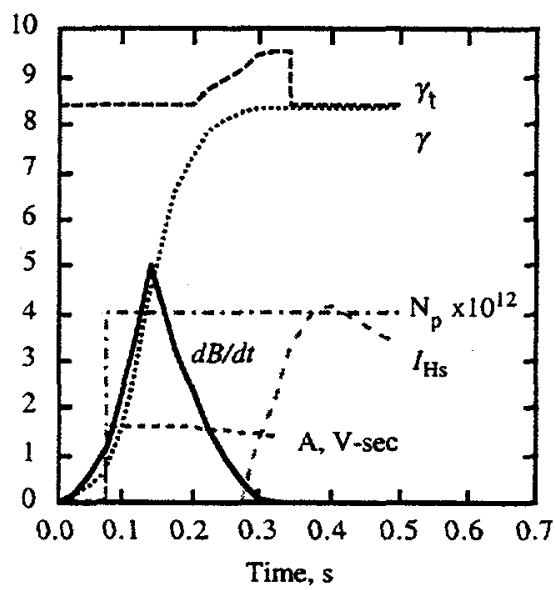

FIGURE 2, The operating mode of the AGS.

\section{EXPERIMENTAL RESULTS}

The transition energy was first measured relative to the gauss clock, which measures the instantaneous field in the dipoles as a function of time, for four values of radial displacement. The published value of $\alpha_{1}$ was checked, by measuring the radial dependence of beam losses as a function of the time at which the phase of the rf accelerating field was flipped to compensate for the reversed direction of shear. The transition energy is obtained from the average of values with the sextupoles on, and is equal to $\gamma_{t}=8.34 \pm 0.05$. The corresponding measurements of $\alpha_{1}$ obtained using equation (4) above give the results $\alpha_{1}=7.2 \pm 1.5 \quad\left(I_{\text {sext, } \mathrm{H}}=0 \mathrm{~A}\right)$ and $\alpha_{1}=3.5 \pm 1.5$ $\left(I_{\text {sex. }, \mathrm{H}}=100 \mathrm{~A}\right)$. These measurements agree roughly with earlier published data however time constraints did not permit good statistics. There may also be a systematic error in the measurement of $\gamma_{1}$ by this method due to the optimum time delay between the phase flip and transition time, because of the nonlinear and asymmetric behavior of the bunches near transition.

When the operating mode described in Figure 2 was implemented, the beam was found to be quite stable near transition. After the first quadrupole synchrotron oscillation all structure tended to damp out and the beam circulated stably without significant losses or beam blowup. Changes in the beam energy, to move below or above transition, or sextupole settings seemed to make little difference and the overall structure of the beam was almost unaffected by accelerator parameters. Losses were not accurately recorded, but seemed small ( $\sim 5 \%)$, and no particular effort was made to minimize them. 


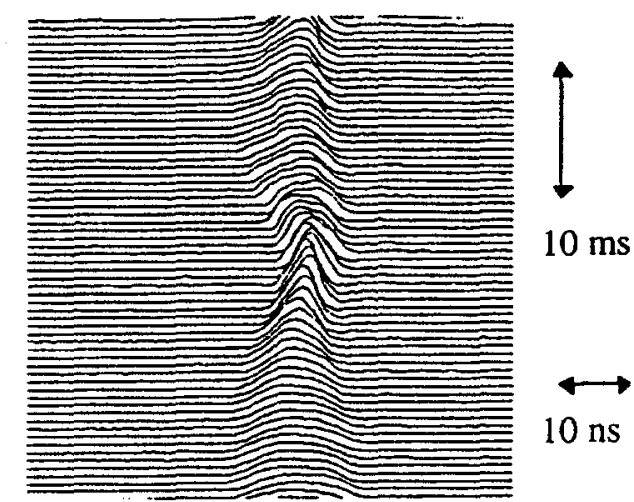

FIGURE 3. Mountain range plot of the bunch after the transition energy was dropped to the beam energy.

The results of bunching tests are shown in Figure 3 and Figure 4, where the bunch shape is plotted both before and after the $\gamma_{\text {t }}$ was suddenly changed. The narrow final shape is noticeably asymmetric because it was impossible to entirely tune out the chromatic effects mentioned above. Runs further from transition showed a number of synchrotron oscillations and the bunch length in later minima was always longer than the first minimum. Closer to transition the bunch length contracted to a minimum and then expanded to roughly the initial value. There seemed to be little emittance blowup. Losses were a function of sextupole currents, but there was insufficient time to explore the dependence in detail. Although the minimum bunch width decreased with increasing sextupole current, the sextuple supplies produced only about half of the current predicted by MAD to optimize $\eta(\delta)$, and we were not able to produce $\alpha_{1}=-1.5$, which is expected to produce the minimum bunch length.

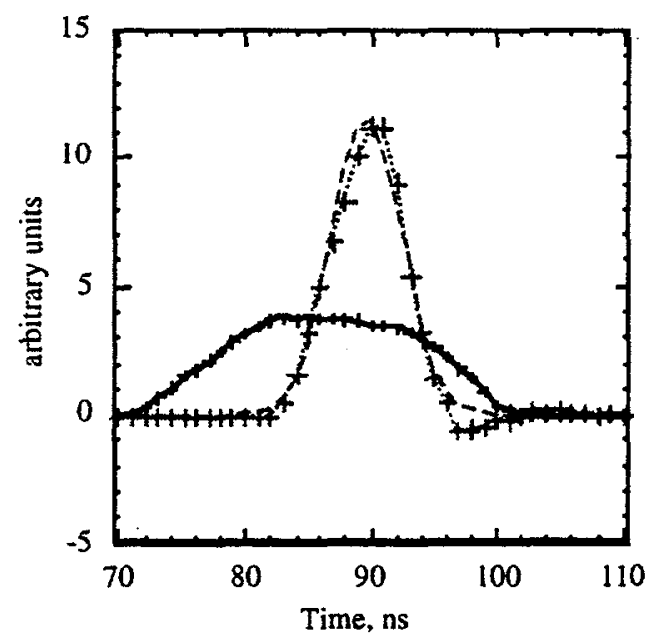

FIGURE 4. Bunch shapes before and after bunching (crosses) showing a final corrected bunch length of $\sigma=2.66 \mathrm{~ns}$ for a $1.5 \mathrm{~V}$-s bunch together with a fitted Gaussian curve (dashed line). 


\section{DISCUSSION}

In order to understand the mechanism of bunching we have done simulations using ESME [10]. These calculations track the longitudinal motion of particles in the bunch over the acceleration and bunching cycle with various parameters. The primary variables are $\Delta \gamma=\gamma_{t}-\gamma$ and $\alpha_{1}$. Simulations show that bunching can occur over a period of $\sim 1 \mathrm{~ms}$ and produce bunches with large momentum spread (full widths of $\delta=$ $0.024)$ and narrow widths. An example of these simulations are shown in Figure 5, (showing $\Delta \mathrm{t}= \pm 15 \mathrm{~ns}, \delta= \pm 0.02$ ), which approximates the parameters shown in Figure 4. These simulations fairly closely match the structure of the data, however the accuracy of the fit seems to be a function of a number of variables such as the parameterization of the density of charge within a bunch. The shape of the shortest bunch that can be produced is sensitive to $\alpha_{1}$ and can be used to estimate this parameter, however the bunch shapes seen do not precisely match those produced in simulations, perhaps indicating higher order terms, $\left(\eta \propto \delta^{2}\right)$. The data seems consistent with $\alpha_{1}=0.0 \pm$ 1.0. The total height of the bunch, $\delta \sim 0.024$, can circulate in the AGS which has a total momentum acceptance of $\delta \sim \pm 0.025$. Since the momentum acceptance is sufficiently large, we expect a further reduction in the bunch width is possible, however this might be accompanied with increased losses if the beams were large.

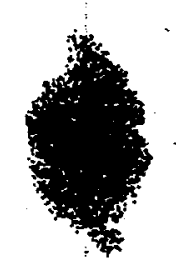

FIGURE 5. Simulation of the final phase space after $\gamma_{l}$ is moved close to the beam energy $(\gamma=8.40)$.

\section{CONCLUSIONS}

During a short run, we were able to explore the operation of the AGS when it was flattopped close to transition. It was found that the bunch length could be reduced by a factor of $\sim 3$ by using the $\gamma_{t}$ jump system to suddenly drop the transition energy to the beam energy, and the beam seemed fairly stable during this exercise. We also measured $\gamma_{\text {}}$ using: 1) the time of phase flip, 2) the synchrotron frequency, and, 3) debunching time, producing rough agreement with previous measurements and with MAD calculations for the lattice. Measurements of $\alpha_{1}$ generally agreed with earlier data (9), but the precision of the data is limited by statistics and measurement time. Attempts to 
tune $\alpha_{1}$ with sextupoles and octupoles were somewhat less successful due to power supply limitations. The stability of the beam circulating near transition was unexpected, and argues for the stability of high current isochronous storage rings.

We were unable to systematically explore possible methods of bunch manipulation due to time constraints, nevertheless, the $\sigma \sim 2.2$ ns bunch length produced by this method is sufficiently short to be useful for the proton driver of the muon collider. A further reduction by $30 \%$ would be even more desirable and this might be obtained by means of the methods tested here. Modification of the bunch shape before rotation, for example, seems useful, but was not optimized. Effects of space charge were also not. studied systematically, and measurements with significant space charge effects would be highly desirable, as would the ability to move the transition energy in two directions, which could be used to produce rapid bunching. A more complete analysis of this data is being published (10).

\section{ACKNOWLEDGEMENTS}

We would like to thank the operating crew of the AGS for their cooperation performing these measurements. We would also like to acknowledge useful discussions with C. Johnstone and D. Neuffer of Fermilab, M. Yoshi and Y. Mori of KEK, and B. Autin of CERN.

\section{REFERENCES}

1. D. Neuffer, Part. Acc. 14 (1983) 75

2. $\mu^{+} \mu$ Collider, A Feasibility Study, published in Proceedings of the Snowmass Workshop, Snowmass 96

3. D. Cline, B. Norum, R. Rossmanith, Proceedings of the European Particle Accelerator Conference, Sitges, Spain, 10-14 Jun 1996. p867

4. M. Brennan, in Proceedings of the Workshop on the Proton Driver for the Muon Collider, Argonne, Nov 14 - 15 1996. Argonne (1996).

5. Lee, Ng and Trbojevic, Phys. Rev. E48 (1993) 3040

6. J. Wei, Longitudinal Dynamics of the Non-Adiabatic Regime on Alternating- Gradient Synchrotrons, Thesis, State University of New York ad Stony Brook, Revised, (1994)

7. H. Grote, F. C. Iselin, The MAD program (methodical accelerator design) : version 8.10; user's reference manual, CERN SL 90-13 AP rev 3. (141 p ). 1993

8. W. K. Van Asselt, L. A. Ahrens, J. M. Brennan, A. Dunber, E. Keith-Monnia, J. T. Morris, M. J. Syphers, Proceedings of the 1995 Particle Accelerator Conference, Dallas, (1995) 3022

9. J. Wei, M. Brennan, L. A. Ahraens, M. M. Blaskiewicz, D-P. Deng, W. W. MacKay. S. Peggs, T. Satogata, D. Trbojevic, A. Werner., W. K. van Asselt, Proceedings of the 1995 Particle Accelerator Conference, Dallas, (1995) 3334

10. J. MacLachlan and J. F. Ostiguy, Proceedings of the 17th IEEE Particle Accelerator Conference (PAC 97) Vancouver, Canada, 12-16 May 1997

11. C. Ankenbrandt, K-Y. Ng, J. Norem, M. Popovic, Z. Qian, L. Ahrens, M. Brennan, V. Mane, T. Roser, D. Trbojevic, W. van Asselt, Fermilab Publication, Fermilab PUB-98-006, (Submitted to Phys Rev D). 